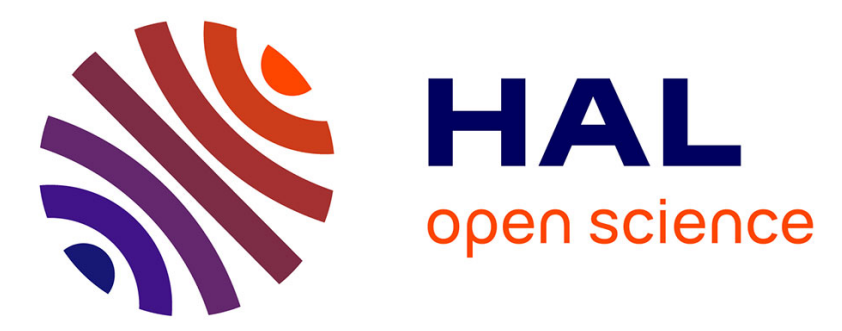

\title{
Best-response team power control for the interference channel with local CSI
}

Paul de Kerret, Samson Lasaulce, David Gesbert, Umer Salim

\section{To cite this version:}

Paul de Kerret, Samson Lasaulce, David Gesbert, Umer Salim. Best-response team power control for the interference channel with local CSI. IEEE International Conference on Communications (ICC 2015), Jun 2015, London, United Kingdom. pp.4132-4136, 10.1109/ICC.2015.7248971 . hal01272506

\section{HAL Id: hal-01272506 https://hal.science/hal-01272506}

Submitted on 15 Feb 2016

HAL is a multi-disciplinary open access archive for the deposit and dissemination of scientific research documents, whether they are published or not. The documents may come from teaching and research institutions in France or abroad, or from public or private research centers.
L'archive ouverte pluridisciplinaire HAL, est destinée au dépôt et à la diffusion de documents scientifiques de niveau recherche, publiés ou non, émanant des établissements d'enseignement et de recherche français ou étrangers, des laboratoires publics ou privés. 


\title{
Best-Response Team Power Control for the Interference Channel with Local CSI
}

\author{
Paul de Kerret*, Samson Lasaulce ${ }^{\S}$, David Gesbert*, and Umer Salim ${ }^{\ddagger}$ \\ * Mobile Communication Department, Eurecom, \{dekerret, gesbert\} @eurecom. fr \\ $\S$ Laboratoire des Signaux et Systèmes (LSS), Supelec, Samson.lasaulce@lss.supelec.fr \\ $\ddagger$ Intel Mobile Communications (IMC), umer.salim@intel.com
}

\begin{abstract}
We study in this paper ${ }^{1}$ the problem of binary power control in interference channels with single-antenna nodes. In many practical scenarios, letting transmitters (TXs) exchange the locally available channel state information (CSI) is unpractical. In such cases, coordinating the power allocation is a difficult problem and we propose in this work a novel binary power control policy for maximizing the ergodic sum-rate when each TX has only access to the instantaneous channel realization of the direct channel to its own user. We prove rigorously the intuitive result that the optimal binary power control policy consists in letting each TX transmits with full power if and only if the realization of this direct channel is above a threshold. The power control policy obtained with the algorithm is a "best-response" power control policy and allows to achieve benefits of coordinated power allocation at a low cost in terms of backhaul resources and complexity.
\end{abstract}

\section{INTRODUCTION}

A large body of literature has focused on the optimization of the power control in wireless communications because of its potential in improving the performance and reducing at the same time the energy consumption [1]-[3]. Maximizing the sum rate in the interference channel (IC) is particularly interesting but is made difficult by its non-convexity. Yet, in the particular case of two single-antennas transmitters (TXs), it is shown in [4] that the binary power control is optimal, thus strongly reducing the complexity of the problem. In the general case with $K$ users, it is verified by simulations that binary power control is an efficient power control scheme.

However, maximizing the sum rate requires the obtaining of the global multi-user channel state information (CSI) at a central node (or at each TX). The cost of this CSI sharing is in many practical settings prohibitive such that approaches running on local CSI have been developed. In particular, this power control problem has then been modeled in several works [5]-[8] as a game theoretic problem where each TX aims at maximizing its own rate. The concavity of the objective is then used to obtain distributed power control algorithms.

This competitive approach comes however with a price in terms of performance reduction, called the price of anarchy [8]. As an answer, we consider the same configuration of only local CSI, but we focus instead on a cooperative approach to maximize the sum rate. Although a common utility is not

\footnotetext{
${ }^{1}$ The research at IMC leading to these results has received funding from the EU FP7 grant iJOIN (No. 317941).
}

intrinsically incompatible with the game theoretic approach, the sum rate utility is no longer concave and has a more complicated structure in terms of the CSI, which makes it impossible to use the above results from the literature. For the particular i.i.d. case, this problem has been considered with an asymptotically large number of users in [9], but no algorithm is provided and the optimality of the thresholding approach is not shown.

Note that the main difficulty that we will face comes from the fact that, following practical considerations, we consider a one-shot optimization problem where no iterative exchange of information between the TXs is allowed. This problem then falls in the category of Bayesian games with incomplete information [10], also called Team Decision [10]-[12] problems when all the users share the same utility. Despite the existence of a rich literature on team decision problems, few solutions have been targeted at wireless scenarios and most techniques assume restrictive properties for the cost function.

Our main contributions read as follows:

- We show that the optimal power control policy consists in having each TX transmit if and only if its channel realization is larger than a given threshold. Hence, finding the optimal binary-power control policy comes down to finding the optimal thresholds.

- In the limiting regimes of high and low SNRs, we provide some optimal thresholds.

- In the general case, we develop an algorithm converging to a best-response power control policy.

\section{System Setting}

\section{A. Transmission Scenario}

We consider an IC consisting of single-antenna nodes with $K$ users and with user $i$ being only served by TX $i$. To make the contributions of this paper as clear as possible, we focus on the 2 -user case i.e., $K=2$. The extension to the $K$-user IC with $K>2$ is ready for almost all the derived results and is briefly discussed in Section V. We denote by $\mathbf{H} \in \mathbb{C}^{2 \times 2}$ the multi-user channel such that the wireless link between TX $j$ and $\mathrm{RX} i$ is equal to $\{\mathbf{H}\}_{i, j}$. We assume further that the transmission occurs in a Rayleigh fast fading environment such that $\{\mathbf{H}\}_{i, j}=\rho_{i, j} \tilde{H}_{i, j}$ with $\tilde{H}_{i, j} \sim \mathcal{N}(0,1)$ being a complex standard Gaussian random variable. The value of $\rho_{i, j}$ reflects 
the geometry (topology) of the network. Finally, we introduce the channel gain matrix $\mathbf{G} \in \mathbb{R}^{2 \times 2}$ where

$$
\{\mathbf{G}\}_{i, j} \triangleq G_{i, j} \triangleq\left|\{\mathbf{H}\}_{i, j}\right|^{2} .
$$

We further consider that the transmitted power $P_{j}$ at TX $j$ is chosen between two power levels $P_{j}^{\min }$ and $P_{j}^{\max }$ with $P_{j}^{\min } \neq P_{j}^{\max }$.

Our main figure of merit in this work will be the ergodic sum-rate with the instantaneous sum-rate being given by [13]

$$
R\left(P_{1}, P_{2}\right)=\log _{2}\left(1+\frac{G_{1,1} P_{1}}{1+G_{1,2} P_{2}}\right)+\log _{2}\left(1+\frac{G_{2,2} P_{2}}{1+G_{2,1} P_{1}}\right) .
$$

\section{B. A Team Decision Problem}

Obtaining accurate global CSI at the TXs is difficult due to the limited feedback and backhaul resources and the changing nature of the wireless channel [14]. As a consequence, we consider that each TX only knows (perfectly) the direct channel to its RX but does not know the realizations of the other channel coefficients. The statistical information, which varies more slowly, is more easily obtained and is supposed to be common knowledge for each TX. Thus, the power control function at TX $j$, which we denote by $p_{j}$, depends only on $G_{j, j}$ and is hence represented as

$$
\begin{array}{rlcc}
p_{j}: & \overline{\mathbb{R}}^{+} & \rightarrow & \left\{P_{j}^{\min }, P_{j}^{\max }\right\} \\
G_{j, j} & \mapsto & p_{j}\left(G_{j, j}\right)
\end{array}
$$

where $\overline{\mathbb{R}}^{+} \triangleq \mathbb{R}^{+} \cup\{\infty\}$. The optimization of the power control function is then written as

$$
\left(p_{1}^{\star}, p_{2}^{\star}\right)=\underset{\left(p_{1}, p_{2}\right) \in \mathcal{P}}{\operatorname{argmax}} \mathbb{E}_{\mathbf{G}}\left[\mathrm{R}\left(p_{1}\left(G_{1,1}\right), p_{2}\left(G_{2,2}\right)\right)\right]
$$

where then notation $\mathbb{E}_{\mathbf{G}}$ indicates that the expectation is taken over $\mathbf{G}$, and $\mathcal{P}$ is defined by

$$
\mathcal{P} \triangleq\left\{\left(p_{1}, p_{2}\right) \mid p_{j}: \overline{\mathbb{R}}^{+} \rightarrow\left\{P_{j}^{\min }, P_{j}^{\max }\right\}, j=1,2\right\} .
$$

Optimization problem (4) is a functional optimization problem, as the optimization is done over the power control functions $p_{j}$. Both TXs aim at maximizing a common utility - the ergodic sum-rate- based on individual information -the knowledge of $G_{j, j}-$, which is a typical Team Decision problem [10]-[12].

Remark 1. It is important to understand that the distributed CSI structure is reflected in the particular dependencies of the power control functions. Hence, the distributed power control problem can be formulated as a centralized optimization problem as long as the optimization variables are functions of the adequate variables.

Solving this functional optimization problem is particularly difficult and we will rely on two main ingredients to simplify the problem at hand. First, leveraging the particular monotonicity properties of the functions considered, we will show that this optimization problem can be cast as a much more simple optimization problem without loss of optimality. The second ingredient consists in using the notion of best-response (BR), which we introduce now, so as to obtain an iterative algorithm (see e.g., [15] [8]).
Definition 1. A best-response power control function is a power control function $\left(p_{1}^{\mathrm{BR}}, p_{2}^{\mathrm{BR}}\right)$ satisfying:

$$
\left\{\begin{array}{l}
p_{1}^{\mathrm{BR}} \in \underset{\left(p_{1}, p_{2}^{\mathrm{BR}}\right) \in \mathcal{P}}{\operatorname{argmax}} \mathbb{E}_{\mathbf{G}}\left[\mathrm{R}\left(p_{1}\left(G_{1,1}\right), p_{2}^{\mathrm{BR}}\left(G_{2,2}\right)\right)\right] \\
p_{2}^{\mathrm{BR}} \in \underset{\left(p_{1}^{\mathrm{BR}}, p_{2}\right) \in \mathcal{P}}{\operatorname{argmax}} \mathbb{E}_{\mathbf{G}}\left[\mathrm{R}\left(p_{1}^{\mathrm{BR}}\left(G_{1,1}\right), p_{2}\left(G_{2,2}\right)\right)\right] .
\end{array}\right.
$$

Intuitively, a best-response power control function is a power control function being optimal given the power control functions at the other TXs. This leads easily to the following well known result [16].

Proposition 1. An optimal power control policy $\left(p_{1}^{\star}, p_{2}^{\star}\right)$ is also a best-response power control policy.

\section{Properties of the Team Decision Problem}

Coming back to an optimal power control policy $\left(p_{1}^{\star}, p_{2}^{\star}\right)$, we provide in this section theoretical results which will be used when designing the power control algorithm in Section IV. As a first step, we introduce a smaller optimization space for the power control functions, namely, the space of threshold functions.

Definition 2. We denote by $\mathcal{T} \subset \mathcal{P}$ the subset containing only the power control functions $\left(p_{1}, p_{2}\right)$ which can be written under the form

$$
p_{j}(x)= \begin{cases}P_{j}^{\min } & \text { if } x \leq \lambda_{j} \\ P_{j}^{\max } & \text { if } x>\lambda_{j}\end{cases}
$$

for some $\lambda_{j} \in \overline{\mathbb{R}}^{+}$. We denote such a power control function by $p_{j}^{\lambda_{j}}$.

Building upon this definition, we can now show the following key property.

Proposition 2. An optimal binary power policy $\left(p_{1}^{\star}, p_{2}^{\star}\right)$ for the optimization problem (4) belongs to $\mathcal{T}$, i.e., there exists a pair $\left(\lambda_{1}^{\star}, \lambda_{2}^{\star}\right) \in \mathbb{R}^{2}$ such that

$$
\left(p_{1}^{\star}, p_{2}^{\star}\right)=\left(p_{1}^{\lambda_{1}^{\star}}, p_{2}^{\lambda_{2}^{\star}}\right) .
$$

Proof. We know from Proposition 1 that an optimal threshold is also a best-response thresholds. Hence, this proposition is shown at the same time as Proposition 4.

Building upon this result, we can obtain an optimal power control policy at asymptotic SNR.

Proposition 3. Let us assume without loss of generality that $P_{1}^{\max }=P_{2}^{\max }=P^{\max }$ and that $\rho_{1,1}^{2} \geq \rho_{2,2}^{2}$. There exists an optimal pair of power control functions $\left(p_{1}^{\star}, p_{2}^{\star}\right)=$ $\left(p_{1}^{\lambda_{1}^{\star}}, p_{2}^{\lambda_{2}^{\star}}\right)$ such that

$$
\lim _{\substack{P_{\max }^{\max } \rightarrow \infty \\ P_{i}^{\min }<\infty}}\left(\lambda_{1}^{\star}, \lambda_{2}^{\star}\right)=(0, \infty)
$$

and

$$
\lim _{P_{\max } \rightarrow 0}\left(\lambda_{1}^{\star}, \lambda_{2}^{\star}\right)=(0,0) .
$$

Proof. A detailed proof is available in the auxiliary document [17]. 
Proposition 2 is especially useful as it shows that solving (4) is equivalent to solving:

$$
\left(\lambda_{1}^{\star}, \lambda_{2}^{\star}\right) \in \underset{\left(\lambda_{1}, \lambda_{2}\right) \in \mathbb{R}^{2}}{\operatorname{argmax}} \mathbb{E}_{\mathbf{G}}\left[\mathrm{R}\left(p_{1}^{\lambda_{1}}\left(G_{1,1}\right), p_{2}^{\lambda_{2}}\left(G_{2,2}\right)\right)\right] .
$$

We now turn to the obtaining of the thresholds $\left(\lambda_{1}^{\star}, \lambda_{2}^{\star}\right)$.

\section{Best Response Power Control Algorithm by BISECTION}

We present here an iterative algorithm where the thresholds of the power control functions are updated in an iterative manner following a "best-response" update. We show first in Subsection IV-A how to update one threshold when the other threshold is kept fixed, and we describe the full algorithm in Subsection IV-B. Note that the proposed algorithm requires to express several conditional expectations and that these calculations are postponed to Subsection IV-C for the sake of clarity.

\section{A. A Characterization of the Optimal Threshold}

Let us consider without loss of generality that the threshold $\lambda_{2}$ is given, i.e., that the power control function at TX 2 is $p_{2}^{\lambda_{2}}$. We start by introducing a new function $\Delta: \overline{\mathbb{R}}^{+} \rightarrow \mathbb{R}$ defined as

$$
\Delta(x) \triangleq \mathbb{E}_{\mathbf{G} \mid G_{1,1}=x}\left[\mathrm{R}\left(P_{1}^{\max }, p_{2}^{\lambda_{2}}\right)\right]-\mathbb{E}_{\mathbf{G} \mid G_{1,1}=x}\left[\mathrm{R}\left(P_{1}^{\min }, p_{2}^{\lambda_{2}}\right)\right]
$$

where then notation $\mathbb{E}_{\mathbf{G} \mid G_{1,1}}$ indicates that the expectation is taken over $\mathbf{G}$ given $G_{1,1}$.

Remark 2. For the sake of clarity, we make a slight abuse of notation by writing $P_{j}^{\min }$ (or $P_{j}^{\max }$ ) to refer to the power control function $p_{j}(x)=P_{j}^{\min }$ (or $P_{j}^{\max }$ ) for all $x$.

This leads to the following result.

Proposition 4. Let the threshold $\lambda_{2}$ be given. There is a unique best-response thresholds $\lambda_{1}^{\mathrm{BR}}$. It is the unique solution of the equation

$$
\Delta(x)=0 .
$$

Proof. By definition of the function $\Delta$ in (12), the bestresponse power control function $p_{1}^{\lambda_{1}^{\mathrm{BR}}}$ necessarily satisfies:

$$
p_{1}^{\lambda_{1}^{\mathrm{BR}}}(x)= \begin{cases}P_{1}^{\min } & \text { if } \Delta(x) \leq 0 \\ P_{1}^{\max } & \text { if } \Delta(x)>0 .\end{cases}
$$

The function $\Delta$ is easily seen to be of class $C^{1}$ and its derivative can be calculated as

$$
\begin{aligned}
\frac{\mathrm{d} \Delta(x)}{\mathrm{d} x} & =\mathbb{E}\left[\frac{P_{1}^{\max }}{1+x P_{1}^{\max }+G_{1,2} P_{2}}-\frac{P_{1}^{\min }}{1+x P_{1}^{\min }+G_{1,2} P_{2}}\right] \\
& =\mathbb{E}\left[\frac{\left(P_{1}^{\max }-P_{1}^{\min }\right)\left(1+G_{1,2} P_{2}\right)}{\left(1+x P_{1}^{\max }+G_{1,2} P_{2}\right)\left(1+x P_{1}^{\min }+G_{1,2} P_{2}\right)}\right]
\end{aligned}
$$

which is strictly positive such that the function $\Delta$ is monotonically increasing. In addition, it can be easily seen that

$$
\Delta(0) \leq 0, \quad \Delta(\infty)>0 .
$$

Using the continuity of $\Delta$, it follows that there is a unique value at which the function $\Delta$ vanishes. By identification with (14), this point is exactly $\lambda_{1}^{\mathrm{BR}}$.

We have hence obtained a useful characterization of the best-response threshold $\lambda_{1}^{\mathrm{BR}}$. In particular, it follows from the monotonicity of $\Delta$ and the fact that it vanishes only at $\lambda_{1}^{\mathrm{BR}}$ that it is possible to use the bisection method to obtain $\lambda_{1}^{\mathrm{BR}}$.

\section{B. Best-Response Power Control Algorithm}

The proposed algorithm consists in applying the bestresponse updating rule for each of the threshold. For clarity, the different steps of the algorithm are put together in Algorithm 1. It remains to prove the convergence of the algorithm.

Proposition 5. Algorithm 1 converges to a best-response power control function $\left(p_{1}^{\mathrm{BR}}, p_{2}^{\mathrm{BR}}\right)$.

Proof. Both TXs optimize a common objective such that this game is a potential game, for which the best-response update is known to converge to a Nash Equilibrium [8].

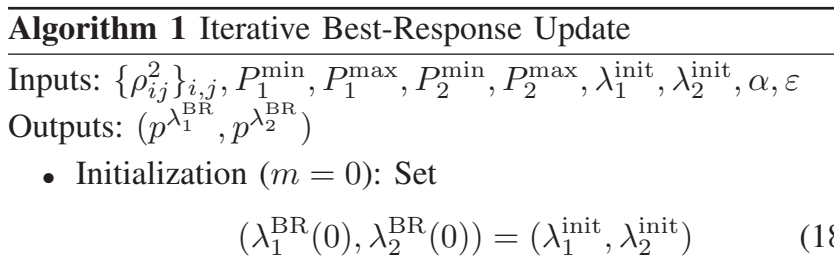

- At step $m \geq 1$ :

1) Update of $P_{1}$ :

- Initialization $(n=0)$ : Set $\lambda_{1}^{\text {low }}(0)=0$ and $\lambda_{1}^{\mathrm{up}}(0)=\alpha \rho_{1,1}^{2}$

- At step $n \geq 1$ : Set

$$
\lambda_{1}^{\mathrm{mid}}(n)=\frac{\lambda_{1}^{\mathrm{low}}(n)+\lambda_{1}^{\mathrm{up}}(n)}{2}
$$

- If $\Delta\left(\lambda_{1}^{\operatorname{mid}}(n)\right) \leq 0$, set

$$
\begin{cases}\lambda_{1}^{\text {low }}(n+1) & =\lambda_{1}^{\text {mid }}(n) \\ \lambda_{1}^{\text {up }}(n+1) & =\lambda_{1}^{\text {up }}(n)\end{cases}
$$

otherwise set

$$
\begin{cases}\lambda_{1}^{\text {low }}(n+1) & =\lambda_{1}^{\text {low }}(n) \\ \lambda_{1}^{\text {up }}(n+1) & =\lambda_{1}^{\text {mid }}(n)\end{cases}
$$

- Proceed until $\left|\lambda_{1}^{\text {up }}(n)-\lambda_{1}^{\text {low }}(n-1)\right|<\varepsilon$. Set $\lambda_{1}^{\text {BR }}=$ $\lambda_{1}^{\operatorname{mid}}(n)$

2) Update of $P_{2}$ : Proceed symmetrically

- Proceed until

$$
\left|\lambda_{1}^{\mathrm{BR}}(m)-\lambda_{1}^{\mathrm{BR}}(m-1)\right|+\left|\lambda_{2}^{\mathrm{BR}}(m)-\lambda_{2}^{\mathrm{BR}}(m-1)\right|<\varepsilon
$$

Remark 3. The proposed algorithm is performed offline, following a designer point of view. It provides power allocation functions which can then be applied in a distributed manner at each TX in the initial Team Decision problem. 


\section{Auxiliary Calculations}

Here, we provide the details on some derivations upon which the results in the preceding sections have been built. More precisely, we calculate the expectations arising in the definition of the function $\Delta$ in (12). The two terms arising in (12) can be calculated in the same manner such that we calculate without loss of generality the conditional expectation for $p_{1}=P_{1}^{\max }$. The expression obtained can then be used for $p_{1}=P_{1}^{\min }$ to evaluate $\Delta$. Furthermore, the same results can be used by symmetry to update the threshold of TX 2 .

We start by calculating the conditional rate of user 1 before turning to the conditional rate of user 2 .

1) $\mathbb{E}_{\mathbf{G} \mid G_{1,1}}\left[R_{1}\left(P_{1}^{\max }, p_{2}\right)\right]$ : Since $p_{2}$ depends only on $G_{2,2}$ it is independent of the channel realizations arising in the expression of $R_{1}$. Hence, it holds that

$$
\begin{aligned}
& \mathbb{E}_{\mathbf{G} \mid G_{1,1}} {\left[R_{1}\left(P_{1}^{\max }, p_{2}\right)\right] } \\
&=\operatorname{Pr}\left(G_{2,2} \leq \lambda_{2}^{\mathrm{BR}}\right) \mathbb{E}_{\mathbf{G} \mid G_{1,1}}\left[R_{1}\left(P_{1}^{\max }, P_{2}^{\min }\right)\right] \quad+\operatorname{Pr}\left(G_{2,2}>\lambda_{2}^{\mathrm{BR}}\right) \mathbb{E}_{\mathbf{G} \mid G_{1,1}}\left[R_{1}\left(P_{1}^{\max }, P_{2}^{\max }\right)\right] \\
&=\left(1-e^{-\frac{\lambda_{2}^{\mathrm{BR}}}{\rho_{2,2}^{2}}}\right) \mathbb{E}_{\mathbf{G} \mid G_{1,1}}\left[R_{1}\left(P_{1}^{\max }, P_{2}^{\min }\right)\right] \\
& \quad+e^{-\frac{\lambda_{2}^{\mathrm{BR}}}{\rho_{2,2}^{2}}} \mathbb{E}_{\mathbf{G} \mid G_{1,1}}\left[R_{1}\left(P_{1}^{\max }, P_{2}^{\max }\right)\right] .
\end{aligned}
$$

Since the power control functions in (24) are only constant functions, we can use the expression for the conditional rate from [18]. This gives for $\left(P_{1}^{\max }, P_{2}^{\max }\right)$

$$
\begin{aligned}
& \mathbb{E}_{\mathbf{G} \mid G_{1,1}}\left[R_{1}\left(P_{1}^{\max }, P_{2}^{\max }\right)\right]=\log \left(1+P_{1}^{\max } G_{1,1}\right) \\
& +e^{\frac{1+P_{1}^{\max } G_{1,1}}{\rho_{1,2}^{2} P_{2}^{\max }}} \mathbb{E}_{1}\left(\frac{1+G_{1,1} P_{1}^{\max }}{\rho_{1,2}^{2} P_{2}^{\max }}\right)-e^{\frac{1}{\rho_{1,2}^{2} P_{2}^{\max }}} \mathbb{E}_{1}\left(\frac{1}{\rho_{1,2}^{2} P_{2}^{\max }}\right)
\end{aligned}
$$

where $\mathbb{E}_{1}$ is the exponential integral function and is defined for $x>0$ as [19, p. 228]

$$
\mathbb{E}_{1}(x) \triangleq \int_{x}^{\infty} \frac{e^{-t}}{t} \mathrm{~d} t .
$$

2) $\mathbb{E}_{\mathbf{G} \mid G_{1,1}}\left[R_{2}\left(P_{1}^{\max }, p_{2}\right)\right]$ : Since $G_{2,2}$ appears in the rate expression of user 2 , we can not use the same calculation as previously and we have to integrate over $G_{2,2}$ :

$$
\begin{aligned}
& \mathbb{E}_{\mathbf{G} \mid G_{1,1}}\left[R_{2}\left(P_{1}^{\max }, p_{2}\right)\right] \\
& =\mathbb{E}_{\mathbf{G}}\left[R_{2}\left(P_{1}^{\max }, p_{2}\right)\right] \\
& =\int_{0}^{\infty} \mathbb{E}_{\mathbf{G} \mid G_{2,2}}\left[R_{2}\left(P_{1}^{\max }, p_{2}\left(G_{2,2}\right)\right] \mathrm{d} G_{2,2}\right. \\
& =\int_{\lambda_{2}^{\mathrm{BR}}}^{\infty} \mathbb{E}_{\mathbf{G} \mid G_{2,2}}\left[R_{2}\left(P_{1}^{\max }, P_{2}^{\max }\right)\right] \mathrm{d} G_{2,2} \\
& \quad+\int_{0}^{\lambda_{2}^{\mathrm{BR}}} \mathbb{E}_{\mathbf{G} \mid G_{2,2}}\left[R_{2}\left(P_{1}^{\max }, P_{2}^{\min }\right)\right] \mathrm{d} G_{2,2} .
\end{aligned}
$$

It remains then to replace the conditional rates inside the two integrals by their expressions using (25). Among the three terms to integrate, two can be easily obtained in closed form but one requires to integrate the exponential integral function $\mathbb{E}_{1}$ defined in (26). Although no closed form could be obtained, this integration can be easily computed with MATLAB. The algebraic manipulations are simple but relatively lengthy and are hence provided in the auxiliary document [17].

Remark 4. In addition, many accurate approximations for the exponential integral exist and can be found in [19, p. 229]. They can then be used to obtain an approximate closed form expression. In [17], the following approximation from [19, p. 229] is used and shown to lead to no noticeable loss over a wide range of parameters:

$$
\frac{1}{2} \log \left(1+\frac{2}{x}\right) \leq e^{-x} \mathbb{E}_{1}(x) \leq \log \left(1+\frac{1}{x}\right) .
$$

\section{EXTENSION TO THE $K$-USER IC}

We have discussed the 2-user IC but all of the results presented in Section III directly generalize to the $K$-user IC with TX $j$ having only instantaneous access to the channel gain $G_{j, j}$ and the statistics of the multi-user channel. In particular, this means that the optimal binary power control functions are also threshold functions, that we can find optimal threshold values tending to zero at low SNR, and that only one TX emits at high SNR. Finally, it is also possible to apply the bisection method to obtain a best-response function, i.e., Algorithm 1 directly extends to $K$ users. The only property which does not easily extend is the possibility to write explicitly the integration because the distribution of the interference is much more complicated in the general case. As a consequence, we will use Monte-Carlo averaging to evaluate the conditional expectations arising in Algorithm 1.

\section{Simulations}

We show in Fig. 1 the ergodic sum-rate attained with the best-response power control function obtained using Algorithm 1 averaged over 10000 Monte-Carlo realizations of the channel. We also choose as parameters for Algorithm 1, $\varepsilon=10^{-3}$ and $\alpha=10$. We consider for the sake of exposition the most simple channel setting with $\rho_{i, j}^{2}=1, \forall i, j$ and $P_{1}^{\max }=P_{2}^{\max }=P^{\max }$ and $P_{1}^{\min }=P_{2}^{\min }=0$. We compare the performance of our algorithm with the two distributed power control policies conventionally used: the egoistic (or competitive) power control scheme where both TXs transmit with full power and the round robin solution where each TX transmits with its maximal power during half the time. Round-robin leads to perfect coordination between the TXs but no opportunistic use of the channel diversity. In addition we also show the performance achieved with perfect CSI at both TXs, which is hence an (a priori) non reachable bound. Finally, we use a linear search to approach the optimal thresholds of the Team Decision problem (4) and not only the best-response optimization problem (5).

It can be seen that the best-response power control outperforms conventional solutions from the literature. It also converges to the optimal solutions at low (egoistic power control) and high SNR (round-robin), respectively. Comparing to the optimal thresholds obtained with the linear search, 
we can see the good match between the two solutions. This confirms that best-response power control policies are a good practical way to approach the optimal power control policies.

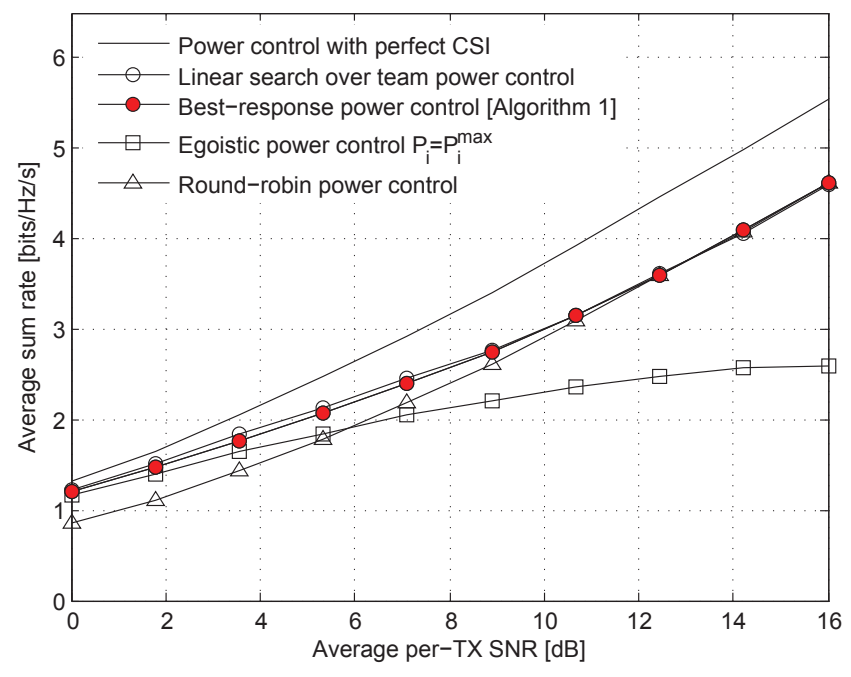

Fig. 1: Average sum-rate as a function of the maximum per-TX transmit power $P^{\max }$ with $K=2$.

Finally, we consider in Fig. 2 the same parameters but with $K=5$. Algorithm 1 is used in combination with Monte-Carlo averaging to evaluate the conditional expectations. It can be seen that the distributed power control policy performs closer to the centralized case than in the two-user case. In the case of i.i.d. Rayleigh fading, it is known that the proposed distributed power control achieves the same scaling in the number of users as centralized power control [9], and we conjecture this property to be also valid for different variance profiles.

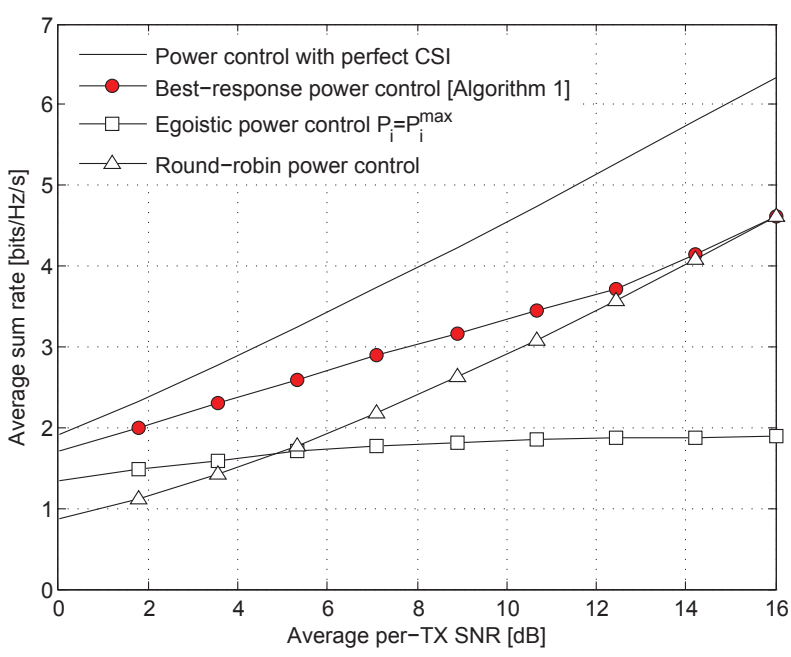

Fig. 2: Average sum-rate as a function of the maximum per-TX transmit power $P^{\max }$ with $K=5$.

\section{CONCLUSION}

We have shown that the binary power control using only local CSI to maximize the sum rate can cast as a Team Decision problem and requires solving a functional optimization problem. Exploiting the particular structure of this problem, we have developed a a simple algorithm converging to a bestresponse power control policy. The proposed algorithm can be computed off-line and once the optimal power control policies have been found, the TXs are only required to compare the channel realizations to the obtained thresholds. Hence, the coordinated power control proposed can be used in settings with low coordination and computation capabilities. Studying the efficiency of the best-response power allocation functions obtained is an interesting and challenging research problem.

\section{REFERENCES}

[1] J. Zander, "Performance of optimum transmitter power control in cellular radio systems," IEEE Trans. Veh. Technol., vol. 41, no. 1, pp. 57-62, Feb 1992.

[2] R. D. Yates, "A framework for uplink power control in cellular radio systems," IEEE J. Sel. Areas Commun., vol. 13, no. 7, pp. 1341-1347, Sep 1995.

[3] S. Stanczak, M. Wiczanowski, and H. Boche, Fundamentals of resource allocation in wireless networks: Theory and algorithms, 2 nd ed. Springer Publishing Company, Incorporated, 2009.

[4] A. Gjendemsjo, D. Gesbert, G. E. Oien, and S. G. Kiani, "Binary power control for sum rate maximization over multiple interfering links," IEEE Trans. on Wireless Commun., vol. 7, no. 8, pp. 3164-3173, 2008.

[5] W. Yu, G. Ginis, and J. M. Cioffi, "Distributed multiuser power control for digital subscriber lines," IEEE J. Sel. Areas Commun., vol. 20, no. 5, pp. 1105-1115, Jun 2002.

[6] G. Scutari, D. P. Palomar, and S. Barbarossa, "Asynchronous iterative water-filling for Gaussian frequency-selective interference channels," IEEE Trans. Inf. Theo., vol. 54, no. 7, pp. 2868-2878, July 2008.

[7] H. Tembine, S. Lasaulce, and M. Jungers, "Joint power controlallocation for green cognitive wireless networks using mean field theory," in Proc. International Conference on Cognitive Radio Oriented Wireless Networks Communications (CROWNCOM), 2010.

[8] S. Lasaulce and H. Tembine, Game theory and learning for wireless networks: Fundamentals and applications, Elsevier, Ed. Academic Press, 2011.

[9] M. Ebrahimi, M. A. Maddah-Ali, and A. K. Khandani, "Throughput scaling laws for wireless networks with fading channels," IEEE Trans. Inf. Theo., vol. 53, no. 11, pp. 4250-4254, Nov. 2007.

[10] J. Marschak and R. Radner, Economic theory of teams. Yale University Press, New Haven and London, Feb. 1972.

[11] R. Radner, "Team decision problems," The Annals of Mathematical Statistics, 1962

[12] Y. C. Ho, "Team decision theory and information structures," Proceedings of the IEEE, vol. 68, no. 6, pp. 644-654, 1980.

[13] T. Cover and A. Thomas, Elements of information theory. WileyInterscience, Jul. 2006.

[14] P. de Kerret and D. Gesbert, "CSI sharing strategies for transmitter cooperation in wireless networks," IEEE Wireless Commun. Mag., vol. 20, no. 1, pp. 43-49, Feb. 2013.

[15] D. Fudenberg and J. Tirole, Game Theory. The MIT Press, 1991

[16] J. Nash, Non-cooperative games. Annals of Mathematics, 1951.

[17] P. de Kerret, S. Lasaulce, D. Gesbert, and U. Salim, "Best-Response Team Power Control for the Interference Channel with Local CSI," 2014, extended version with detailed calculation. Available online in arXiv.

[18] M. C. Filippou, D. Gesbert, and G. A. Ropokis, "Optimal combining of instantaneous and statistical CSI in the SIMO interference channel," in Proc. IEEE Vehicular Technology Conference (VTC Spring), 2013.

[19] M. Abramowitz and I. A. Stegun, Handbook of mathematical functions, 10th ed. Dover Publications Inc., New York, 1972. 